\title{
Toxicity evaluation of nanocrystalline silver-impregnated coated dressing on the life cycle of worm Caenorhabditis elegans
}

\author{
A. Ayech ${ }^{a}$, M.E. Josende ${ }^{a}$, J. Ventura-Lima ${ }^{a}$, C. Ruas ${ }^{b}$, M.A. Gelesky ${ }^{b}$, A. Ale ${ }^{c}$, J. Cazenave $^{c}$, \\ J.M. Galdopórpora ${ }^{\mathrm{d}}$, M.F. Desimone ${ }^{\mathrm{d}}$, M. Duarte ${ }^{\mathrm{a}}$, P. Halicki ${ }^{\mathrm{e}}$, D. Ramos ${ }^{\mathrm{e}}$, L.M. Carvalho ${ }^{\mathrm{f}}$, \\ G.C. Leal ${ }^{\mathrm{f}}$, J.M. Monserrat ${ }^{\mathrm{a}, *}$
}

a Universidade Federal do Rio Grande- FURG, Instituto de Ciências Biológicas (ICB), Programa de Pós-graduação em Ciências Fisiológicas (PPGCF), Rio Grande, RS, Brazil ${ }^{\mathrm{b}}$ Universidade Federal do Rio Grande- FURG, Escola de Química e Alimentos (EQA), Programa de Pós-graduação em Química Tecnológica e Ambiental (PPGQTA), Rio Grande, RS, Brazil

${ }^{\mathrm{c}}$ Instituto Nacional de Limnología (INALI), UNL, CONICET, Santa Fe, Argentina

${ }^{\mathrm{d}}$ Universidad de Buenos Aires (UBA), CONICET, Instituto de Química y Metabolismo del Fármaco (IQUIMEFA), Facultad de Farmacia y Bioquímica, Buenos Aires, Argentina

${ }^{\mathrm{e}}$ Universidade Federal do Rio Grande- FURG, Faculdade de Medicina (FAMED), Programa de Pós-graduação em Ciências da Saúde (PPGCS), Rio Grande, RS, Brazil

${ }^{\mathrm{f}}$ Universidade Federal de Santa Maria (UFSM), Departamento de Química, Programa de Pós-graducação em Ciências Farmacéuticas, Santa María, RS, Brazil

\section{A R T I C L E I N F O}

\section{Keywords:}

Nanotechnology

Antibacterial

Reactive oxygen species

Toxicity

Silver nanoparticles

\begin{abstract}
A B S T R A C T
In recent times, however, due to the emergence of bacterial strains with resistance to conventional antibiotics, silver has again gained attention as an alternative for developing new efficient bactericides, including the use of silver nanoparticles (AgNPs). However, the improper disposal of these items after use may cause toxicological effects on organisms in the environment. To evaluate the potential environmental hazard of nanosilver-coated dressings, the nematode Caenorhabditis elegans was chosen as a test organism. The assays were conducted in 24well plates that contain four different sizes of coated dressing to obtain different concentrations. L1 and L4 C. elegans larval stages were exposed to these nanosilver concentrations. Dressing cutouts were arranged between two layers of agar for 3 days and Escherichia coli (OP 50 strain) was added as food source for the worms. After the exposure period, growth, reproduction, fertility, silver concentration in the medium and the concentration of reactive oxygen species (ROS) in the worms were evaluated. Scanning and transmission electron microscopy analyses were performed on the coated dressings, as well as analyses of zeta potential, ionic release and antibacterial power in two bacterial strains (Pseudomonas aeruginosa and Staphylococcus aureus). It was verified the antibacterial power of the coated dressing, in both bacteria strains tested. Characterization of the coated dressing indicated heterogeneous nanoparticles, as well as distinct zeta potentials for the medium in water and saline medium $(0.9 \% \mathrm{NaCl})$. L1 larval worms exposed to nanosilver-coated dressing showed a high ROS concentration and reductions in growth, fertility and reproduction. Worms exposed to the coated dressing during the L4 stage showed almost no response. Overall, the obtained results indicate the potential environmental hazard of nanosilver-coated dressings.
\end{abstract}

\section{Introduction}

The antimicrobial properties of silver are well-known, indeed its use in wound disinfection has been documented since the 18th century (Klasen, 2000). Silver nitrate $\left(\mathrm{AgNO}_{3}\right)$ has been widely used in the treatment of ulcers but, with the discovery of penicillin, its application as bactericide has declined (Klasen, 2000; Chopra, 2007). In recent times, however, due to the emergence of bacterial strains with resistance to conventional antibiotics, silver has again gained attention as an alternative for developing new efficient bactericides (Chopra, 2007; Gurunathan et al., 2014). Based on the same evidence, the development of new nanotechnologies has led to an increase in the use of silver nanoparticles (AgNPs). There is a range of materials that use nanosilver as a raw material, including wound coated dressings, surgical instruments and many other goods and products (Bosetti et al., 2002; Cohen et al., 2007; Chen and Schluesener, 2008; Paladini and

\footnotetext{
${ }^{*}$ Corresponding author. Universidade Federal do Rio Grande - FURG, Instituto de Ciências Biológicas (ICB). Av Itália km 8 s/n - Caixa Postal 474, 96200-970, Rio Grande, RS, Brazil.

E-mail addresses: monserrat_jm@furg.br, josemmonserrat@gmail.com (J.M. Monserrat).
} 
Pollini, 2019; Kalantari et al., 2020). The currently available coated dressings contain varying silver concentration and include products like Acticoat (Smith and Nephew) and Actisorb (Johnson and Johnson) (Silver et al., 2006). Silver, as a antibacterial, is used in various products; the AgNPs impregnate the surfaces of them, like coated dressing, leading to the release of silver ions $\left(\mathrm{Ag}^{+}\right)$. This release is associated with the antimicrobial efficacy (Wijnhoven et al., 2009).

Smith and Nephew manufactures the Acticoat Flex coated dressing. It markets ACTICOAT ${ }^{\circledR}$ FLEX 3 (registered in Brazil by national agency ANVISA through number 80804050025) and ACTICOAT ${ }^{\circledR}$ FLEX 7, with differences in the maximum silver concentration $\left(1.64 \mathrm{mg} / \mathrm{cm}^{2}\right.$ and $2.0 \mathrm{mg} / \mathrm{cm}^{2}$, respectively). ACTICOAT ${ }^{\circledast}$ FLEX 3 and ACTICOAT ${ }^{\circledR}$ FLEX 7 act as active antimicrobial barrier for at least 3 and 7 days, respectively (Smith \& Nephew Medical Ltd.)

Like other nanoparticles, AgNPs exhibit toxicity related to the size/ surface relationship, where the smaller the particle, the greater its toxicity. Smaller particles more easily cross cell membranes and, because of their high relative surface, interaction with organelles is easier (Liu et al., 2010; Wang et al., 2012; Gliga et al., 2014). The entry of nanoparticles into cells occurs either through the plasma membrane (diffusion or membrane channels) or endocytosis (AshaRani et al., 2009; Singh et al., 2009). Once inside, molecules accumulate outside the mitochondria, and promote reactive oxygen species (ROS) generation. AgNPs can also interact with organelles to cause oxidative damage to proteins, membranes, and DNA. Another possible mechanism involves the interaction of nanoparticles and silver ions in $\mathrm{Ca}^{2+}$ release in the cytoplasm. This release triggers cell signalling cascades that activate catabolic enzymes including phospholipases, proteases, and endonucleases, all of which damage the mitochondrial membranes and cytoskeleton that can culminate in programmed cell death (Orrenius et al., 1992, 2015).

The namatode Caenorhabditis elegans is widely applied in experimental studies due to its short life cycle, small size and easy cultivation. It is also as it is very sensitive to environmental changes (Hope, 1999; Leung et al., 2008; Rodriguez et al., 2013). The worm feeds on bacteria, especially living or dead Escherichia coli (Liu et al., 2012). This species was, in fact, one of the selected test organisms in the NanoReg initiative (NanoReg, 2016). Based on data from the scientific literature cited above, this study aimed to evaluate the potential toxicity of the ACTICOAT FLEX 3 coated dressing on the model organism C. elegans.

\section{Materials and methods}

\subsection{Coated dressings acquisition}

Acticoat Flex 3 coated dressings from Smith and Nephew were purchased via the internet. To perform the tests, there were used the coated dressings of lots 1715, 1647, 1616, and 1819.

\subsection{Observation of silver crystals by scanning electron microscopy}

Small cutouts of the coated dressing were dried by the Autosamdri815 (Sample Drying at the critical point) and then visualized by a HighLow Low-Vacuum Scanning Electron Microscope (SEM), Jeol, JSM $6610 \mathrm{LV}$, with EDS probe to analyze the presence of silver on the surface of the coated dressing fibers. With the aid of the free image editing software ImageJ, the crystals were measured and obtained the frequency size distribution.

\subsection{Observation of silver crystals by transmission electron microscopy}

Pieces of the coated dressing were placed in 2 different solutions, one with MilliQ water and the other in $0.9 \% \mathrm{NaCl}$ saline solution. After 3 days, drops of these solutions were transferred to carbon grids for electron microscopy for $10 \mathrm{~min}$ and then were placed on filter paper to dry for $24 \mathrm{~h}$. The analyses were performed with a Jeol JEM-
$1400120 \mathrm{keV}$ Transmission Electron Microscope, coupled with an EDS probe.

\subsection{Zeta potential measurement}

Pieces of the coated dressing were inserted into deionised water and $0.9 \%$ saline over $3 \mathrm{~d}$, and the solutions were sonicated at $10 \%$ of the total $400 \mathrm{~W}$ power of the Bonitech Branson sonicator and then analyzed on the equipment (Litesizer 500).

\subsection{Ionic release of silver from nanoparticles}

To perform the release of $\mathrm{Ag}^{+}$ions from silver dressing, four pieces (each one of $1 \mathrm{~cm}^{2}$ ) were included in $15 \mathrm{~mL}$ of distilled water. An aliquot of $0.5 \mathrm{~mL}$ of media was replaced at each time $(1,2,6,12,24,36$, $48,60,72 \mathrm{~h}$ ). Aliquots were evaluated using a VGP 210 atomic absorption spectrophotometer (BuckScientific, East Norwalk, CT, USA) by electrothermal atomization using pyrolytic graphite furnace.

\subsection{Silver concentration in the coated dressing}

To obtain the total silver concentration contained in the coated dressings at $\mathrm{cm}^{2}$ of the coated dressing, four pieces of coated dressing $\left(1 \mathrm{~cm}^{2}\right)$ were mineralized using $9 \mathrm{~mL}$ of nitric acid (96\%) and $1 \mathrm{~mL}$ of hydrogen peroxide (100\%). The mixture was then heated using a micro digester (mls 1200 mega). The silver content was measured by atomic absorption spectroscopy as described above.

\subsection{Silver in the NGM medium}

The Nematode Growth Medium (NGM) that was used to wrap the coated dressing was removed after 3 days and placed in an eppendorf tube and stored in a freezer. After that, samples were melted at $90{ }^{\circ} \mathrm{C}$ and analyzed by atomic absorption spectroscopy, as described previously.

\subsection{Antibacterial power of coated dressing}

To evaluate the antimicrobial potential of Acticoat flex 3 against Pseudomonas aeruginosa (ATCC 15442) and Staphylococcus aureus (ATCC 12598), an agar diffusion-based method was used as follows: $15 \mathrm{ml}$ of Mueller Hinton agar medium was added to a Petri dish $(90 \times 15 \mathrm{~mm})$; after solidification of the medium, a MacFarland 0.5 bacterial suspension $\left(1.5 \times 10^{8} \mathrm{UCF} / \mathrm{mL}\right)$ of each strain was prepared and plated onto the entire surface of the medium using a swab. Then a portion of the circular impregnated bandage $\left(50 \mathrm{~mm}\right.$ in diameter, $1.64 \mathrm{mg} / \mathrm{cm}^{2}$ ) was placed centrally on the surface of the culture medium containing the seeded bacteria. The plates were incubated at $37^{\circ} \mathrm{C}$ for $24 \mathrm{~h}$ and, after the diameter of the inhibition zone was measured. The experiment was performed in triplicate.

For the determination of the susceptibility profile of the evaluated strains, a second batch of experiments was carried out with antibiotics, where $P$. aeruginosa was exposed to Ciprofloxacin $(5 \mu \mathrm{g})$, Ceftazidime $(30 \mu \mathrm{g})$, Gentamicin $(10 \mu \mathrm{g})$, Piperacycline-Tazobactam (100mcg$10 \mathrm{mcg})$, and Meropenem (10 $\mu \mathrm{g})$, and $S$. aureus to Ciprofloxacin $(5 \mu \mathrm{g})$, Penicillin (10U), and Tetracycline $(30 \mu \mathrm{g})$. The tests and the interpretation of the results were performed following the Performance Standards for Antimicrobial Susceptibility Testing protocol (CLSI M100, 2017).

\subsection{Animal model and maintenance}

Animals of strain N2, Bristol (wild strain) were cultured in Petri cell culture (medium and large) with nematode-appropriate NGM culture medium (NGM: nematode growth media) (3.0 g of NaCl/L; $5.0 \mathrm{~g}$ of peptone/L; $5.0 \mathrm{mg}$ of cholesterol/L, $1 \mathrm{mmol}$ of $\mathrm{CaCl}_{2} / \mathrm{L} ; 1 \mathrm{mmol}$ of 
$\mathrm{MgSO}_{4} / \mathrm{L} ; 25 \mathrm{mmol}$ of $\mathrm{KH}_{2} \mathrm{PO}_{4} / \mathrm{L}$; and $17 \mathrm{~g}$ of agar/L diluted in $1 \mathrm{~L}$ of autoclaved Milli Q water at $\mathrm{pH} 6.0$ after preparation) and kept under temperature-controlled by incubator set at $20{ }^{\circ} \mathrm{C}$. The plates were seeded with Escherichia coli bacteria, non-pathogenic strain OP50, with optical density 1.0 at $600 \mathrm{~nm}$, as a food source for $C$. elegans, according to the traditional maintenance procedures described by Brenner (1974).

\subsection{Preparation of animals for experiments}

To develop the experiment, it was necessary to obtain a population of synchronized animals with the same larval stage. Ovate animals were removed by washing the culture plates using $\mathrm{M} 9$ buffer $\left(3.0 \mathrm{~g} \mathrm{KH}_{2} \mathrm{PO}_{4}\right.$, $6 \mathrm{~g} \mathrm{Na}_{2} \mathrm{HPO}_{4}, 5 \mathrm{~g} \mathrm{NaCl}$ and $1 \mathrm{~mL} 1 \mathrm{M} \mathrm{MgSO}_{4}$ diluted in $1 \mathrm{~L}$ of sterilized Milli Q water, $\mathrm{pH}$ 6) using Pasteur pipettes and then accommodated in $50 \mathrm{~mL}$ Falcon tubes for centrifugation $1000 \mathrm{~g}$ for $10 \mathrm{~min}$, at $20{ }^{\circ} \mathrm{C}$ and pellet formation of animals and disposal of supernatant containing NGM and $E$. coli residues. Then, the bleaching procedure was performed using a caustic solution ( $\mathrm{NaOH} 10 \mathrm{M}$ and $2.5 \%$ sodium hypochlorite) in which the ovated animals are lysed, releasing their fertilized eggs. At the end of this procedure only the eggs remain alive, being separated by centrifugation ( $150 \mathrm{~g}$ for $15 \mathrm{~min}$ at $20{ }^{\circ} \mathrm{C}$ with a $30 \%$ sucrose solution). Finally, the eggs were transferred to Petri dishes containing M9 medium and incubated at $20{ }^{\circ} \mathrm{C}$ for $14 \mathrm{~h}$ until the animals hatched and reached their first stage of larval development (L1). The entire synchronization procedure follows the protocol proposed by Stiernagle (2006), which was originally formulated to remove fungal and bacterial contamination from C. elegans cultures.

\subsection{Plate preparation}

All the experiments were performed in triplicate using true independent replicates. To assemble the plates, the coated dressings were cut in circles with a $1 \mathrm{~cm}$-diameter and placed in a 24-well plate. To obtain different coated dressing concentrations, it was assumed that the silver concentration was uniform over the entire coated dressing area. The following silver concentrations were selected: $1.64 \mathrm{mg} / \mathrm{cm}^{2}$ (whole puddle equivalent $=1 / 1$ or $100 \%$ ), $1.23 \mathrm{mg} / \mathrm{cm}^{2}$ (three-quarter puddle equivalent $=3 / 4$ or $75 \%$ ), $0.82 \mathrm{mg} / \mathrm{cm}^{2}$ (half-puddle equivalent $=1 / 2$ or $50 \%$ ), and $0.41 \mathrm{mg} / \mathrm{cm}^{2}$ (quarter-puddle equivalent $=1 / 4$ or $25 \%$ ) and CTRL for control group with animals exposed only to NGM and $E$. coli OP50 (Figs. 1 and 2 of Supplementary Material).

CTRL for animals exposed to coated dressings that were released for 6 days, 3 days releasing on the agar, after the agar is removed and the coated dressing washed with autoclaved Milli Q water, after they were put back in the agar for 3 more days. The mounting of the exposure plate was performed in a sterile environment by applying a first NGM film $(500 \mu \mathrm{L})$. Afterward, the coated dressing cutouts were introduced into each one of the 24-well plates and covered with another layer of NGM $(500 \mu \mathrm{L})$, thus forming two films around the coated dressing (Fig. 1 of Supplementary Material). Then the plate was incubated at $37^{\circ} \mathrm{C}$, simulating human body temperature for 3 days, another group of plates was incubated at $20^{\circ} \mathrm{C}$, also for 3 days. This time was selected as this is indicated by the manufacturer for material release, with the bottom of the plate facing up so that the silver released by the coated dressing could be available to the animals on the surface of the NGM during the exposure period. It was observed a darker coloration in the NGM that was in contact with the cuttings that had a larger size (higher concentrations). At the end of the third day of incubation, the exposure plates were removed from the $37{ }^{\circ} \mathrm{C}$ incubator and cooled to $20{ }^{\circ} \mathrm{C}$ (exposure temperature). Immediately after, $50 \mu \mathrm{L}$ of $E$. coli OP50 was pipetted as a food source. Following, C. elegans in larval stage L1 (an average of 20 animals per puddle) were introduced into the puddles.

The average number of animals introduced into each pool was reached by estimating 10 aliquots with a volume of $10 \mu \mathrm{L}$ of a C. elegans suspension in the liquid medium, which was performed by counting. The total number of animals among all aliquots was divided by the estimated aliquots to determine the volume to be pipetted into each pool at the time of exposure (Solis and Petrascheck, 2011).

After incubation, the plates were kept in an incubator at $20{ }^{\circ} \mathrm{C}$ for $96 \mathrm{~h}$, the necessary time for the animals to complete their developmental cycle through the larval stages L1, L2, L3, and L4 and reach the adult stage, so they are able to grow and reproduce, giving rise to the first progeny (Hope, 1999). Then, their evaluation of growth, fertility rate, and reproduction was performed.

In the case of animal exposure in L4, the plate assembly procedures were repeated, with incubation modifications, where it occurred only at $20{ }^{\circ} \mathrm{C}$ (both during the dressing release period and during the 3-day exposure), using only a pool with a nominal concentration of $1.64 \mathrm{mg}$ / $\mathrm{cm}^{2}$ (100\%).

All the analyses performed in this work were based on the international standard ISO 10872: 2010 adopted as reference for the toxicity tests evaluated by NANoREG. For the analysis, photos were taken of the puddles, using a magnifying glass $(80 \times)$ coupled with a camera (Leica, model S8 APO). All analyses were performed with the help of free image editing software ImageJ. For better visualization of the animals in the evaluation of growth, reproduction, and fertility, the photo acquisition procedures were performed after staining of the animals with Rose Bengal dye.

\subsection{Growth assessment}

After taking the pictures of the adult animals stained with Rose Bengal, the subjects were measured with the ImageJ software. The growth rate (in $\mathrm{mm}$ ) was measured by subtracting adult animal size from L1 larval stage animal size.

\subsection{Fertility assessment}

In order to calculate the fertility percentage, the presence or absence of eggs within the body of adult animals was observed individually. An animal was considered pregnant (fertile) if the number of eggs within its body is $\geq 1$. After counting the number of pregnant hermaphrodite worms and subtracting the number of male worms from the total adult animals, the fertility percentage was calculated by dividing the number of fertile animals by the number of adult animals and multiplying by 100 .

\subsection{Reproduction assessment}

To proceed with the reproduction calculation, the number of L1 larval stage animals counted in each pool was divided by the number of adult fertile animals in the respective pool. Results were expressed as the number of larvae per exposed adult.

\subsection{Reactive oxygen species concentration (ROS) dosage}

It was performed via fluorescence microscopy using the whole worm exposed to the probe $\mathrm{H}_{2}$ DCF-DA (SIGMA), according to Büchter et al. (2013). After $1 \mathrm{~h}$-incubation, photos were taken with the Dino-Lite microscope (AM4115T-GFBW) at $200 \times$ of magnification, using 480 and $510 \mathrm{~nm}$ for excitation and emission, respectively. Subsequently, using the ImageJ software, the area of the animals was measured, together with fluorescence intensity and, around the animals, the background was also measured and the following expression calculated: $\mathrm{ROS}=(\mathrm{FI}-\mathrm{BG}) / \mathrm{A}$, where FI stands for worm fluorescence intensity, BG for background and A for the animal area.

\subsection{Statistics}

Data were expressed as mean \pm 1 standard error of the mean (SEM). L1 worm growth, reproduction, fertility, and ROS concentration data were analyzed by a mixed model analysis of variance (ANOVA), 

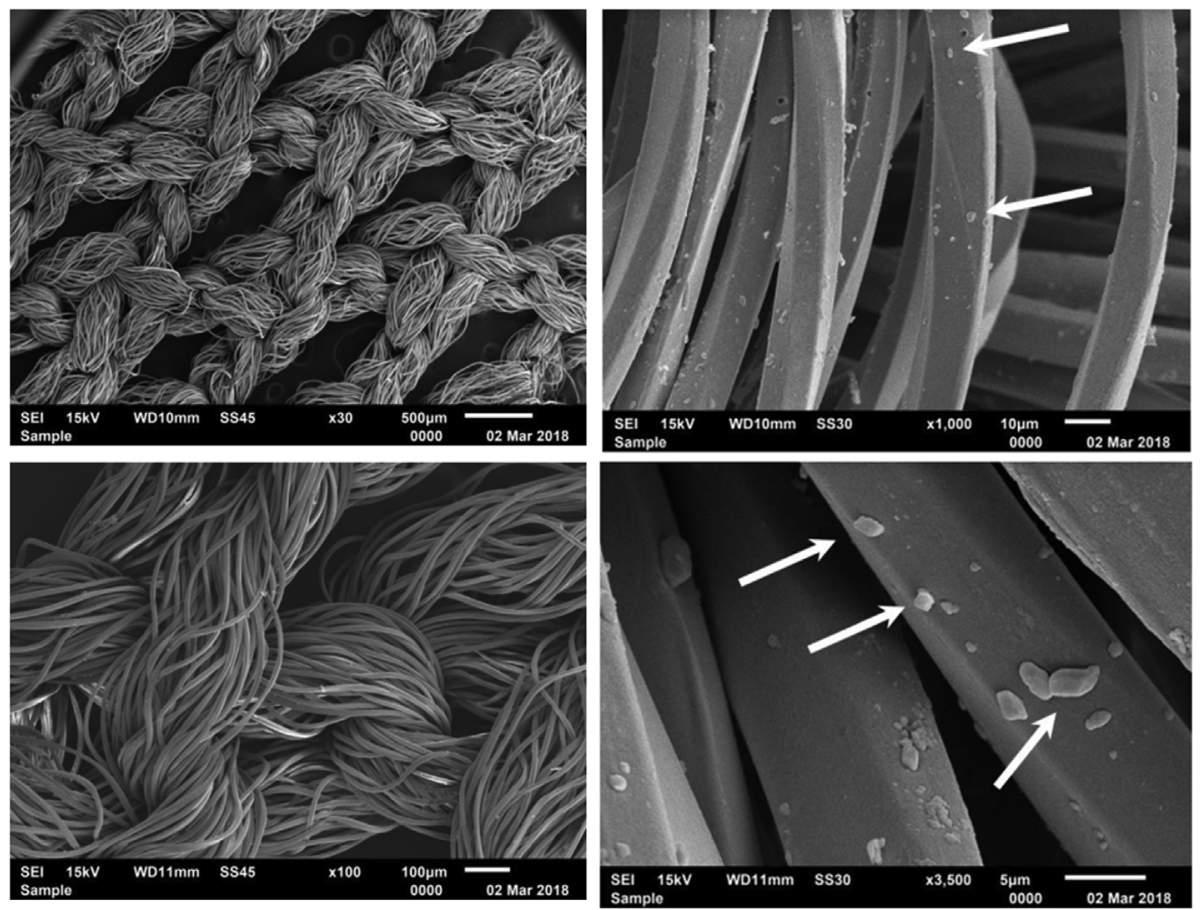

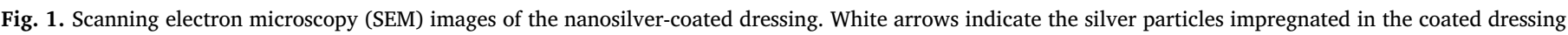
fibers.

being the random factor, the different plates employed in the independent experiments and fixed the factor was nanosilver concentration (Searle et al., 2006). Previously, normality and homoscedasticity assumptions were evaluated through Shapiro-Wilks and Levene tests, respectively, and mathematical transformations applied if at least one of these was violated (Zar, 1984). The same procedure was employed to analyze L4 worms data. Statistical differences among means treatment were performed using the post-hoc test of Newman-Keuls. In all cases, a significance level $(\alpha)$ of 0.05 was adopted.

\section{Results and discussion}

\subsection{Physico-chemical results}

\subsubsection{Characterization of silver-coated dressing}

Through SEM analysis, it was possible to observe the structure of the dressing with the entangled polyester fibers (Fig. 1a and b) and particles randomly dispersed and impregnated in the dressing fibers (Fig. 1c and d). To perform a size distribution analysis, silver nanoparticles from the coated dressing samples were analyzed through TEM, after immersion in saline solution ( $0.9 \% \mathrm{NaCl}$; Fig. $2 \mathrm{a}$ and b) or MilliQ water (Fig. 2c and d). From the EDS analysis performed on the ACTICOAT FLEX 3 coated dressing, it was possible to detect the following compounds: silver (75.4\%), oxygen (18.5\%) and carbon (5.78\%) (Fig. 3 of Supplementary Material).

The coated dressings immersed in the $0.9 \%$ saline solution showed greater agglomeration than the ones immersed in Mili $Q$ water. Measurements performed with ImageJ software demonstrated that the particles immersed in the $0.9 \%$ saline solution had a larger size (mean of c.a. $80 \mathrm{~nm}$ ) than the particles that were immersed in MilliQ water (mean of c.a. $40 \mathrm{~nm}$ ), which were much less crowded (Figs. 3 and 4). Reports including Doty et al. (2005) and Michaels et al. (2000) revealed that high $\mathrm{NaCl}$ concentrations cause AgNPs to aggregate. The zeta potential of the nanoparticles immersed in $0.9 \%$ saline solution averaged $-17.8 \mathrm{mV}$, whereas those immersed in deionised water averaged -25 $\mathrm{mV}$. Some guidelines show that zeta potential values between \pm 20-30 mV are moderately stable (Patel and Agrawal, 2011;
Bhattacharjee, 2016). Thus, the obtained results indicated that the nanoparticles released by the coated dressing in saline solution have a tendency to be less stable than those in deionised water. Interestingly, the manufacturer's use instructions state that the coated dressing must be moistened in potable water and not in saline, an advisory that once again corroborates our results.

AgNP toxicity towards bacteria was reported in several studies (Lok et al., 2006; Choi et al., 2008). Pal et al. (2007) evaluated the effects of bactericidal activity in different exposure media. The authors observed that exposure in agar presents greater toxicity compared to liquid medium. The use of silver is advantageous when compared to other classes of antibiotics because microorganisms, in general, do not show resistance to this compound (Romero-Urbina et al., 2015; Sangappa and Thiagarajan, 2015). Baker et al. (2005) and Panácek et al. (2006) presented data on the antimicrobial capacity of silver against $E$. coli and Staphylococcus aureus. Baker et al. (2005) found that particles with a higher surface-to-volume ratio provide a more efficient means for antibacterial activity against E. coli, and Panácek et al. (2006) found similar results, where $25 \mathrm{~nm}$ sized-AgNPs show greater antimicrobial and bactericidal activities compared to larger particles including against highly multidrug-resistant strains such as methicillin-resistant $S$. aureus. Mohan et al. (2014) also reported a high antimicrobial activity of AgNPs against Pseudomonas aeruginosa. The AgNP antibacterial capacity was also observed in the present work, is that there is evidence that silver diffuses in the medium and that there is an antimicrobial action against these two microorganisms that we evaluated, because there is a halo of inhibition (Tables 1 and 2 and Figs. 4-6 of Supplementary Material).

\subsubsection{Ionic release of silver}

The total silver content of the dressing was $1.44 \mathrm{mg} / \mathrm{cm}^{2}$. The coated dressing analyzed in this study presented a controlled silver release over time. Indeed, $34 \%$ of the total silver content of the dressing was released after $72 \mathrm{~h}$ (Fig. 4). It is worth to mention that the release of the bactericide agent is required to achieve antimicrobial activity in wound dressings (Mebert et al., 2016). AgNP have the ability to release silver ions, and this property contributes to the bactericidal effect 



Fig. 2. Transmission electron microscopy (TEM) images of the nanosilver-coated dressing immersed in saline solution ( $0.9 \% \mathrm{NaCl}$; a and b) or MilliQ water (c and d).

already provided by silver (Morones et al., 2005). In recent work using C. elegans, it was found that also the shape of AgNP (nanoparticles, nanowires and nanoplates) influences toxicity (Moon et al., 2019).

\subsection{Toxicity evaluation in C. elegans}

\subsubsection{Reactive oxygen species concentration (ROS) dosage}

The Fenton reation, ion release and ROS generation occur on the surface of nanoparticles, thus very small molecules $(<30 \mathrm{~nm}$ ) have a relatively large surface area and then, also have higher toxic effects than the larger ones (Auffan et al., 2009). With a highly variable particle distribution, as seen in Figs. 2 and 3, it can be inferred that at a concentration of $100 \%$ there should provide enough small nanoparticles to induce higher ROS concentration in worms, as shown in Fig. 5. The obtained results agree with Yang et al. (2018), who found high ROS concentration in C. elegans exposed to commercial AgNPs and explained that oxidative stress is the main toxic mechanism generated by these particles. The augmented ROS concentrations could affect the metabolism of energy in C. elegans, since oxidative chemicals as in this case results to be the coated dressing, generate mitochondrial malfunction, and consequently, the oxidative stress in the cytoplasm may increase inducing cell apoptosis (Luo et al., 2017).

\subsubsection{Physiologic parameters}

Kim et al. (2017) evaluated the effect of AgNPs on C. elegans growth and reported that concentrations of $0.01 \mathrm{mg} / \mathrm{L}$ already induces significant differences, reducing size. Data from Contreras et al. (2014) showed that concentrations between 1 and $10 \mathrm{mg} \mathrm{AgNPs/L} \mathrm{significantly}$ reduce in $C$. elegans growth, however, after continuous exposure through several generations, growth return to normal. Then, a pattern of acclimatisation was evidence when worms are exposed to $100 \mathrm{mg}$ AgNP/L. The C. elegans fertility is also affected by AgNPs, where smaller particles induce the greatest toxicity effects. Roh et al. (2009) provided data where concentrations of $0.1 \mathrm{mg} \mathrm{AgNPs/L}$ impairs C. elegans reproduction, and reduces the number of offspring by up to $70 \%$ after a $24 \mathrm{~h}$-exposure. In this study, we verified that all evaluated physiological parameters were compromised when the organisms were exposed to the coated dressing (Fig. 5). The data from in this study corroborate those already found in the literature, where exposure to silver nanoparticles also reduced the size of adults and reduced fertility and reproduction of worm C. elegans.

Stage L4 was less prone to show toxic effects after nanosilver-coated dressing exposure than the L1 (Table 1). The obtained results may indicate that stage L4 is more resistant than the initial L1 larval stage. Chaweeborisuit et al. (2016) exposed all C. elegans larval stages to plumbagin (a nematicide). These authors reported that L4 is least sensitive, while L1 shows the highest sensitivity. Chu and Chow (2002) also observed that L1 animals are more susceptible when exposed to different cadmium concentrations than animals at more advanced stages. Overall, the use of the L1 stage offers greater sensitivity to observe potential deleterious effects of chemical stressors. C. elegans has a cuticle that is a layered internal structure with surface specializations and is known to undergo changes in its composition and structure during the life stages of the worm (Kramer, 1997). Probably the permeability in L4 is lower due to its thicker cuticle (Chaweeborisuit et al., 2016).

As previously mentioned, to obtain different exposure concentrations, the dressings were cut into different sizes. The data showed that there was high variability among same-sized dressings with regards to the silver concentrations released into the NGM, this can be easily observed by the high standard deviation of the samples (Fig. 7 of Supplementary Material), especially in the $100 \%$ cut $\left(1.64 \mathrm{mg}\right.$ silver $\left./ \mathrm{cm}^{2}\right)$. This finding could indicate that the silver is not homogenously distributed in the coated dressing.

The dressings that released their contents for 3 days in the NGM, were washed and added to a new NGM medium, and they still released silver, as verified by atomic absorption spectroscopy $(211.33 \pm 33.64 \mu \mathrm{g} / \mathrm{g})$. However, no toxic effects were observed in worms exposed to these dressing (See Table 3 of Supplementary Material). 




(b)

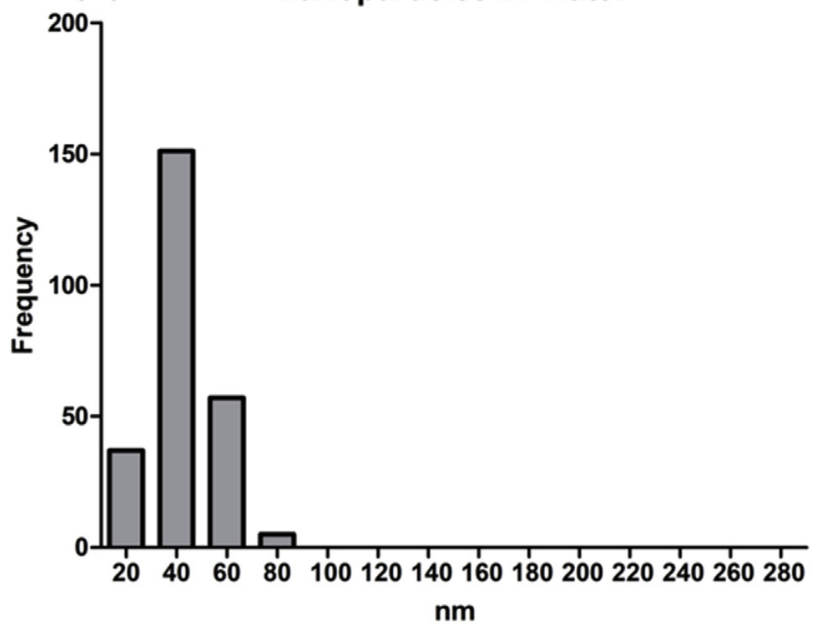

(c)

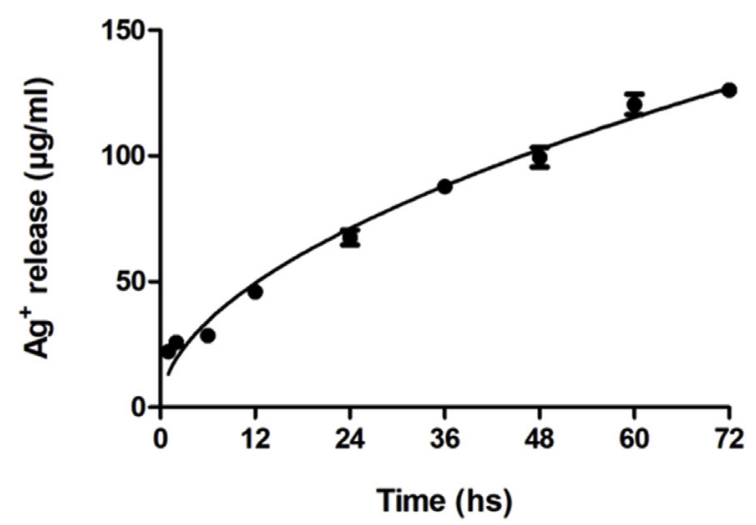

Fig. 3. The frequency size distribution of nanosilver-coated dressing immersed in $0.9 \% \mathrm{NaCl}$ saline solution (a) or in MilliQ water (b). Silver (Ag) release versus time, expressed in concentration units $(\mathrm{mg} / \mathrm{ml})(\mathrm{c})$.
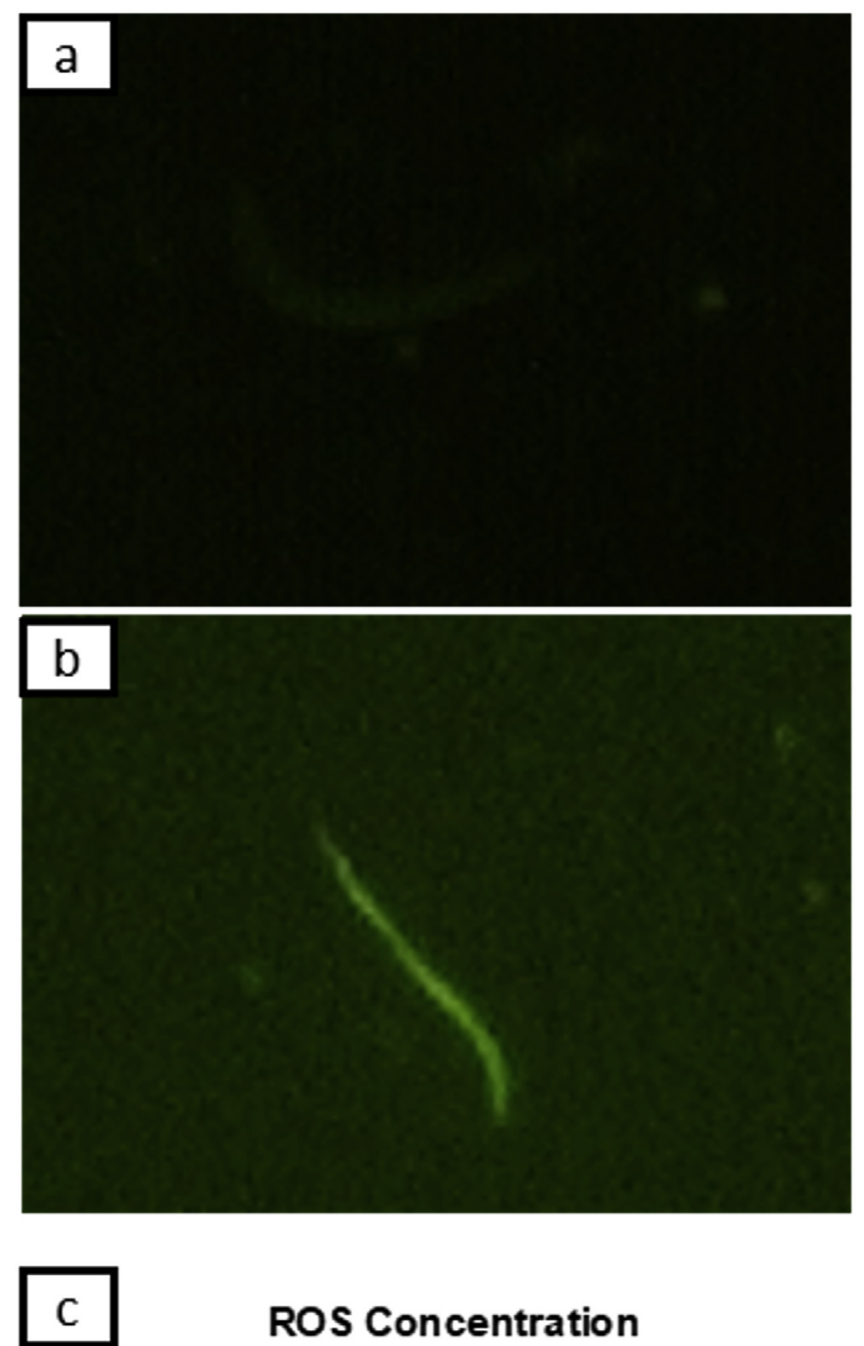

\section{ROS Concentration}



Fig. 4. Reactive oxygen species (ROS) concentration measured through fluorescence emission using the $\mathrm{H}_{2}$ DCF-DA probe, where greater fluorescence intensity indicates a higher ROS concentration. (a) Caenorhabditis elegans exposed to a coated dressing with $1.64 \mathrm{mg}$ silver $/ \mathrm{cm}^{2}$ at stage L1. (b) C. elegans from control group. (c) Fluorescence intensity in C. elegans stage L1 for the different treatments: $1.64 \mathrm{mg}$ silver $/ \mathrm{cm}^{2}$ (whole puddle equivalent $=1 / 1$ or $100 \%$ ), $1.23 \mathrm{mg}$ silver $/ \mathrm{cm}^{2}$ (three-quarter puddle equivalent $=3 / 4$ or $75 \%$ ), $0.82 \mathrm{mg}$ silver $/ \mathrm{cm}^{2}$ (half-puddle equivalent $=1 / 2$ or $50 \%$ ), $0.41 \mathrm{mg}$ silver $/ \mathrm{cm}^{2}$ (quarter-puddle equivalent $=1 / 4$ or $25 \%$ ) and the control group (Ctrl). Values are expressed as mean +1 standard error. Equal letters indicate the absence of significant differences ( $\mathrm{p}>0.05$ ). 
(a)

Growth



(b)

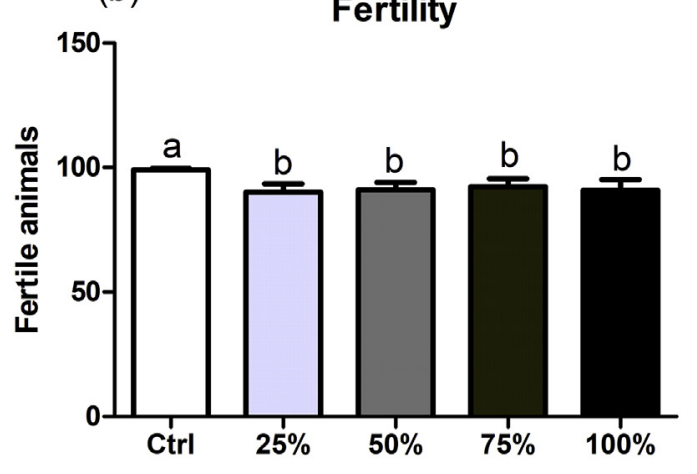

(c)

\section{Reproduction}

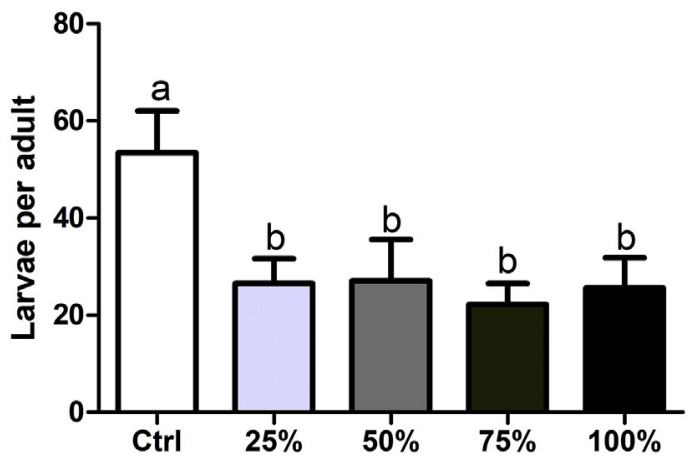

Fig. 5. Physiological responses in Caenorhabditis elegans after 3-day exposure to the following treatments: $1.64 \mathrm{mg}$ silver $/ \mathrm{cm}^{2}$ (whole puddle equivalent $=1 / 1$ or $100 \%$ ), $1.23 \mathrm{mg}$ silver $/ \mathrm{cm}^{2}$ (three-quarter puddle equivalent $=3 / 4$ or $75 \%$ ), $0.82 \mathrm{mg}$ silver $/ \mathrm{cm}^{2}$ (half-puddle equivalent $=1 / 2$ or $50 \%$ ), $0.41 \mathrm{mg}$ silver $/ \mathrm{cm}^{2}$ (quarter-puddle equivalent $=1 / 4$ or $25 \%$ ) or control (Ctrl). Values are expressed as mean +1 standard error for growth (a), fertility (b), and (c) number of larvae generated ( $n=4-5$ for each treatment). Equal letters indicate the absence of significant differences $(p>0.05)$.

\section{Conclusions}

It was observed that in water the particles were less agglomerated and more stable as verified in zeta potential, unlike the saline medium $(0.9 \% \mathrm{NaCl})$, where the particles were more agglomerated, which is expected situation under those physiological conditions. Results indicated that Acticoat Flex 3 has an antibacterial effect as do antibiotics in the strains tested ( $P$. aeruginosa and $S$. aureus). Nanosilver-coated dressing impaired reduced reproduction, growth and fertility and induced higher ROS concentration in L1 larval stage animals, unlike L4
Table 1

Evaluation of the physiological parameters of L4 larval stage worms.

\begin{tabular}{lll}
\hline L4 Stage & \multicolumn{2}{l}{} \\
\hline Physiological parameters & \multicolumn{2}{l}{ Mean and Standard Error } \\
\cline { 2 - 3 } & Control & $100 \%$ \\
\hline Growth (mm) & $1.05 \pm 0.01^{\mathrm{a}}$ & $1.05 \pm 0.02^{\mathrm{a}}$ \\
Fertility (\%) & $96.67 \pm 1.76^{\mathrm{a}}$ & $98.99 \pm 1.01^{\mathrm{a}}$ \\
Reproduction (larvae/adult) & $41.52 \pm 0.72^{\mathrm{a}}$ & $36.15 \pm 2.30^{\mathrm{a}}$ \\
ROS Concentration & $33670.94 \pm 1730.08^{\mathrm{a}}$ & $33882.73 \pm 984.47^{\mathrm{a}}$
\end{tabular}

$100 \%$ refers to worms exposed to $1.64 \mathrm{mg} / \mathrm{cm} 2$ (whole puddle equivalent $=1$ / 1 or $100 \%$ ) for 3 days. Growth is expressed in millimeters, fertility is expressed as a percentage of fertile animals, reproduction expressed by the ratio of larvae to adult animals. ROS is expressed by the fluorescence intensity using the $\mathrm{H}_{2}$ DCF-DA probe. Values are expressed as mean +1 standard error. Equal letters indicate no significant differences $(\mathrm{p}>0.05)$ for each physiological variable.

stage animals, which showed lower susceptibility or no changes in the measured parameters Finally, the obtained results suggest that improper disposal of this coated dressing has the potential to cause damage to organisms, including $C$. elegans, and this finding highlights its environmental hazard.

\section{CRediT authorship contribution statement}

A. Ayech: Conceptualization, Methodology, Formal analysis, Investigation, Writing - original draft, Writing - review \& editing, Visualization. M.E. Josende: Conceptualization, Methodology, Investigation, Writing - original draft, Writing - review \& editing, Visualization. J. Ventura-Lima: Conceptualization, Resources, Writing - original draft, Writing - review \& editing. C. Ruas: Validation, Writing - review \& editing, Visualization. M.A. Gelesky: Validation, Resources, Writing - review \& editing, Visualization, Funding acquisition. A. Ale: Conceptualization, Writing - original draft, Writing - review \& editing. J. Cazenave: Writing - original draft, Writing - review \& editing. J.M. Galdopórpora: Validation, Formal analysis, Investigation, Writing original draft, Writing - review \& editing, Visualization. M.F. Desimone: Validation, Resources, Writing - original draft, Writing review \& editing, Visualization, Funding acquisition. M. Duarte: Investigation, Writing - original draft, Writing - review \& editing. P. Halicki: Validation, Investigation, Writing - original draft, Writing review \& editing. D. Ramos: Validation, Resources, Writing - original draft, Writing - review \& editing, Funding acquisition. L.M. Carvalho: Validation, Writing - original draft, Writing - review \& editing, Funding acquisition. G.C. Leal: Validation, Investigation, Writing - original draft, Writing - review \& editing. J.M. Monserrat: Conceptualization, Methodology, Validation, Formal analysis, Resources, Writing - original draft, Writing - review \& editing, Visualization, Project administration, Funding acquisition.

\section{Declaration of competing interest}

The authors declare that there are no conflicts of interest.

\section{Acknowledgments}

This study was financed in part by the Coordenação de Aperfeiçoamento de Pessoal de Nível Superior - Brasil (CAPES) Finance Code 001. J.M. Monserrat is a productivity fellow from Brazilian CNPq (process number PQ 308539/2016-8, respectively).

\section{Appendix A. Supplementary data}

Supplementary data to this article can be found online at https:// 
doi.org/10.1016/j.ecoenv.2020.110570.

\section{References}

AshaRani, P., Hande, M.P., Valiyaveettil, S., 2009. Anti-proliferative activity of silver nanoparticles. BMC Cell Biol. 10 (1), 65. https://doi.org/10.1186/1471-2121-10-65.

Auffan, M., Rose, J., Bottero, J., Lowry, G.V., Jolivet, J., Wiesner, M.R., 2009. Towards a definition of inorganic nanoparticles from an environmental, health and safety perspective. Nat. Nanotechnol. 4, 634-641. https://doi.org/10.1038/nnano.2009.242.

Baker, C., Pradhan, A., Pakstis, L., Pochan, D.J., Shah, I., 2005. Synthesis and antibacterial properties of silver nanoparticles. J. Nanosci. Nanotechnol. 5 (2), 244-249. https://doi.org/10.1166/jnn.2005.034.

Bhattacharjee, S., 2016. DLS and zeta potential - what they are and what they are not? J. Contr. Release 235, 337-351. https://doi.org/10.1016/j.jconrel.2016.07.002. 2016.

Bosetti, M., Massè, A., Tobin, E., Cannas, M., 2002. Silver coated materials for external fixation devices: in vitro biocompatibility and genotoxicity. Biomaterials 23 (3), 887-892. https://doi.org/10.1016/s0142-9612(01)00198-3.

Brenner, S., 1974. The genetics of Caenorhabditis elegans. Genetics 77 (1), 71-94.

Büchter, C., Ackermann, D., Havermann, S., Honnen, S., Chovolou, Y., Fritz, G., Kampkötter, A., Wätjen, W., 2013. Myricetin-mediated lifespan extension in Caenorhabditis elegans is modulated by DAF-16. Int. J. Mol. Sci. 14 (6), 11895-11914. https://doi.org/10.3390/ijms140611895.

Chaweeborisuit, P., Suriyonplengsaeng, C., Suphamungmee, W., Sobhon, P., Meemon, K., 2016. Nematicidal effect of plumbagin on Caenorhabditis elegans: a model for testing a nematicidal drug. Zeitschrift fur Naturforschung C71, 121-131. https://doi.org/10. 1515/znc-2015-0222.

Chen, X., Schluesener, H.J., 2008. Nanosilver: a nanoproduct in medical application. Toxicol. Lett. 176 (1), 1-12. https://doi.org/10.1016/j.toxlet.2007.10.004.

Choi, O., Deng, K.K., Kim, N.-J., Ross, L., Surampalli, R.Y., Hu, Z., 2008. The inhibitory effects of silver nanoparticles, silver ions, and silver chloride colloids on microbial growth. Water Res. 42 (12), 3066-3074. https://doi.org/10.1016/j.watres.2008.02 021.

Chopra, I., 2007. The increasing use of silver-based products as antimicrobial agents: a useful development or a cause for concern? J. Antimicrob. Chemother. 59 (4), 587-590. https://doi.org/10.1093/jac/dkm006.

Chu, K.W., Chow, K.L., 2002. Synergistic toxicity of multiple heavy metals is revealed by a biological assay using a nematode and its transgenic derivative. Aquat. Toxicol. 61 (1-2), 53-64. https://doi.org/10.1016/s0166-445x(02)00017-6.

CLSI, 2017. Performance Standards for Antimicrobial Susceptibility Testing. 27th Ed. CLSI Supplement M100. Clinical and Laboratory Standards Institute, Wayne, PA.

Cohen, M.S., Stern, J.M., Vanni, A.J., Kelley, R.S., Baumgart, E., Field, D., Libertino, J.A., Summerhayes, I.C., 2007. In vitro analysis of a nanocrystalline silver-coated surgical mesh. Surg. Infect. 8 (3), 397-404. https://doi.org/10.1089/sur.2006.032.

Contreras, E.Q., Puppala, H.L., Escalera, G., Zhong, W., Colvin, V.L., 2014. Size-dependent impacts of silver nanoparticles on the lifespan, fertility, growth, and locomotion of Caenorhabditis elegans. Environ. Toxicol. Chem. 33 (12), 2716-2723. https://doi. org/10.1002/etc. 2705 .

Doty, R.C., Tshikhudo, T.R., Brust, M., Fernig, D.G., 2005. Extremely stable water-soluble Ag nanoparticles. Chem. Mater. 17 (18), 4630-4635. https://doi.org/10.1021/ cm0508017.

Gliga, A.R., Skoglund, S., Odnevall Wallinder, I., Fadeel, B., Karlsson, H.L., 2014. Sizedependent cytotoxicity of silver nanoparticles in human lung cells: the role of cellular uptake, agglomeration and Ag release. Part. Fibre Toxicol. 11 (1), 11. https://doi. org/10.1186/1743-8977-11-11.

Gurunathan, S., Han, J., Kwon, D.N., Kim, J.H., 2014. Enhanced antibacterial and antibiofilm activities of silver nanoparticles against Gram-negative and Gram-positive bacteria. Nanoscale Research Letters 9 (1), 373. https://doi.org/10.1186/1556-276x9-373.

Hope, I.A., 1999. Instructions for Use ACTICOAT FLEX. Oxford University Press, NY. https://www.smith-nephew.com/global/assets/pdf/products/brasil/2016-04/ 80804050025-acticoatflex-ifu0025-revb.pdf, Accessed date: 12 September 2019.

Kalantari, K.1, Mostafavi, E.2, Afifi, A.M.3, Z4, Izadiyan, Jahangirian, H.2, RafieeMoghaddam, R.2, Webster TJ2Katayoon Kalantari, K., Mostafavi, E., Afifi, A.M., Izadiyan, Z., Jahangirian, H., Rafiee-Moghaddam, R., Webster, T.J., 2020. Wound dressings functionalized with silver nanoparticles: promises and pitfalls. Nanoscale 12 (4), 2268-2291. https://doi.org/10.1039/c9nr08234.

Kim, J.H., Lee, S.H., Cha, Y.J., Hong, S.J., Chung, S.K., Park, T.H., Choi, S.S., 2017. C. elegans-on-a-chip for in situ and in vivo Ag nanoparticles' uptake and toxicity assay. Sci. Rep. 7 (40225), 1-11. https://doi.org/10.1038/srep40225.

Klasen, H.J., 2000. Historical review of the use of silver in the treatment of burns. I. Early uses. Burns 26 (2), 117-130. https://doi.org/10.1016/s0305-4179(99)00108-4.

Kramer, J.M., Riddle, D.L., et al., 1997. In: Cold Spring Harbor Laboratory Press, pp. 471-500.

Leung, M.C.K., Williams, P.L., Benedetto, A., Au, C., Helmcke, K.J., Aschner, M., Meyer, J.M., 2008. Caenorhabditis elegans: an emerging model in biomedical and environmental toxicology. Toxicol. Sci. 106, 5-28. https://doi.org/10.1093/toxsci/kfn121.

Liu, H., Wang, X., Wang, H.-D., Wu, J., Ren, J., Meng, L., Wu, Q., Dong, H., Wu, J., Kao, T., Ge, Q., Wu, Z., Yuh, C., Shan, G., 2012. Escherichia coli noncoding RNAs can affect gene expression and physiology of Caenorhabditis elegans. Nat. Commun. 3 (1). https://doi.org/10.1038/ncomms2071.

Liu, W., Wu, Y., Wang, C., Li, H.C., Wang, T., Liao, C.Y., Cui, L., Zhou, Q.F., Yan, B., Jiang, G.B., 2010. Impact of silver nanoparticles on human cells: effect of particle size. Nanotoxicology 4 (3), 319-330. https://doi.org/10.3109/17435390.2010.483745.

Lok, C., Ho, C., Chen, R., He, Q., Yu, W., Sun, H., Tam, P.K., Chiu, J., Che, C., 2006
Proteomic analysis of the mode of antibacterial action of silver nanoparticles. J. Proteome Res. 5, 916-924. https://doi.org/10.1021/pr0504079.

Luo, X., Xu, S., Yang, Y., Zhang, Y., Wang, S., Chen, S., Xu, A., Wu, L., 2017. A novel method for assessing the toxicity of silver nanoparticles in Caenorhabditis elegans. Chemosphere 168, 648-657. https://doi.org/10.1016/j.chemosphere.2016.11.011.

Mebert, A.M., Aimé, C., Alvarez, G.S., Shi, Y., Flor, S.A., Lucangioli, S.E., Desimone, M.F., Coradin, T., 2016. Silica core-shell particles for the dual delivery of gentamicin and rifamycin antibiotics. J. Mater. Chem. B 4, 3135. https://doi.org/10.1039/ C6TB00281A.

Michaels, A.M., Jiang, Brus, L., 2000. Ag nanocrystal junctions as the site for surfaceenhanced Raman scattering of single rhodamine 6G molecules. J. Phys. Chem. B 104 (50), 11965-11971. https://doi.org/10.1021/jp0025476.

Mohan, S., Oluwafemi, O.S., George, S.C., Jayachandran, V.P., Lewu, F.B., Songca, S.P., Kalarikkal, N., Thomas, S., 2014. Completely green synthesis of dextrose reduced silver nanoparticles, its antimicrobial and sensing properties. Carbohydr. Polym. 106, 469-474. https://doi.org/10.1016/j.carbpol.2014.01.008.

Moon, J., Kwak, J.I.I., Na, Y., 2019. The effects of silver nanomaterial shape and size on toxicity to Caenorhabditis elegans in soil media. Chemosphere. http://doi.org/10. 1016/j.chemosphere.2018.09.177.

Morones, J.R., Elechiguerra, J.L., Camacho, A., Holt, K., Kouri, J.B., Ramírez, J.T., Yacaman, M.J., 2005. The bactericidal effect of silver nanoparticles. Nanotechnology 16 (10), 2346-2353. https://doi.org/10.1088/0957-4484/16/10/059.

NANoREG, November 2016. A Common European Approach to the Regulatory Testing of Nanomaterials. Final Report (part 1). updated 2017/02/21.

Orrenius, S., Gogvadze, V., Zhivotovsky, B., 2015. Calcium and mitochondria in the regulation of cell death. Biochem. Biophys. Res. Commun. 460, 72-81. https://doi. org/10.1016/j.bbrc.2015.01.137.

Orrenius, S., McCabe, M.J., Nicotera, P., 1992. $\mathrm{Ca}^{2+}$-dependent mechanisms of cytotoxicity and programmed cell death. Toxicol. Lett. 64-65, 357-364. https://doi.org/ 10.1016/0378-4274(92)90208-2.

Pal, S., Tak, Y.K., Song, J.M., 2007. Does the antibacterial activity of silver nanoparticles depend on the shape of the nanoparticle? A study of the gram-negative bacterium Escherichia coli. Appl. Environ. Microbiol. 73 (6), 1712-1720. https://doi.org/10. 1128/AEM.02218-06.

Paladini, F., Pollini, M., 2019. Antimicrobial silver nanoparticles for wound healing application: progress and future trends. Materials 12, 2540. https://doi.org/10.3390/ ma12162540.

Panácek, A., Kvítek, L., Prucek, R., Kolár, M., Vecerová, R., Pizúrová, N., Sharma, V.K., Nevecná, T., Zboril, R., 2006. Silver colloid nanoparticles: synthesis, characterization, and their antibacterial activity. J. Phys. Chem. B 110 (33), 16248-16253. https://doi. org/10.1021/jp063826h.

Patel, V.R., e Agrawal, Y.K., 2011. Nanosuspension: an approach to enhance solubility of drugs. "J. Adv. Pharm. Technol. Research"“ (JAPTR)" 2 (2), 81-87. https://doi.org/ 10.4103/2231-4040.82950.

Rodriguez, M., Snoek, L.B., De Bono, M., Kammenga, J.E., 2013. Worms under stress: $C$. elegans stress response and its relevance to complex human disease and aging. Trends Genet. 29, 367-374. https://doi.org/10.1016/j.tig.2013.01.010.

Roh, J., Sim, S.J., Yi, J., Park, K., Chung, K.H., Ryu, D., Choi, J., 2009. Ecotoxicity of silver nanoparticles on the soil nematode Caenorhabditis elegans using functional ecotoxicogenomics. Environ. Sci. Technol. 43 (10), 3933-3940. https://doi.org/10.1021/ es803477u.

Romero-Urbina, D.G., Lara, H.H., Velázquez-Salazar, J.J., Arellano-Jiménez, M.J., Larios, E., Srinivasan, A., Lopez-Ribot, J.L., Yacamán, M.J., 2015. Ultrastructural changes in methicillin-resistant Staphylococcus aureus induced by positively charged silver nanoparticles. Beilstein J. Nanotechnol. 6, 2396-2405. https://doi.org/10.3762/ bjnano.6.246.

Sangappa, M., Thiagarajan, P., 2015. Combating drug resistant pathogenic bacteria isolated from clinical infections, with silver oxide nanoparticles. Indian J. Pharmaceut. Sci. 77, 151-155. https://doi.org/10.4103/0250-474X.156546.

Searle, S.R., Casella, G., 2006. McCulloch. Hoboken, New Jersey.

Silver, S., Phung, L.T., Silver, G., 2006. Silver as biocides in burn and wound dressings and bacterial resistance to silver compounds. J. Ind. Microbiol. Biotechnol. 33 (7), 627-634. https://doi.org/10.1007/s10295-006-0139-7.

Singh, N., Manshian, B., Jenkins, G.J.S., Griffiths, S.M., Williams, P.M., Maffeis, T.G.G., Wright, C.J., Doak, S.H., 2009. NanoGenotoxicology: the DNA damaging potential of engineered nanomaterials. Biomaterials 30 (23-24), 3891-3914. https://doi.org/10. 1016/j.biomaterials.2009.04.009.

Solis, G.M., Petrascheck, M., 2011. Measuring Caenorhabditis elegans life span in 96 well microtiter plates. JoVE 49. https://doi.org/10.3791/2496.

Stiernagle, T., 2006. Wormbook 11, 1-11. https://doi.org/10.1895/wormbook.1.101.1.

Wang, Z., Chen, J., Li, X., Shao, J., Peijnenburg, W.J.G.M., 2012. Aquatic toxicity of nanosilver colloids to different trophic organisms: contributions of particles and free silver ion. Environ. Toxicol. Chem. 31 (10), 2408-2413. https://doi.org/10.1002/etc. 1964.

Wijnhoven, S.W.P., Peijnenburg, W.J.G.M., Herberts, C.A., Hagens, W.I., Oomen, A.G., Heugens, E.H.W., Roszek, B., Bisschops, J., Goses, I., Dik Van De Meent, D.V., Dekkers, S., De Jong, W.H., Van Zijverden, M., Sips, A.J.A.M., Geertsma, R.E., 2009. Nano-silver - a review of available data and knowledge gaps in human and environmental risk assessment. Nanotoxicology 3 (2), 109-138. https://doi.org/10. 1080/17435390902725914.

Yang, Y., Xu, G., Xu, S., Chen, S., Xu, A., Wu, L., 2018. Effect of ionic strength on bioaccumulation and toxicity of silver nanoparticles in Caenorhabditis elegans. Ecotoxicol. Environ. Saf. 165, 291-298. https://doi.org/10.1016/j.ecoenv.2018.09. 008.

Zar, J.H., 1984. Englewood Cliffs. Prentice-Hall, NJ. 